\title{
Klinische Muster des Körperausdrucks bei Schmerz
}

\author{
Joseph Walsh
}

Schmerzpatienten artikulieren ihre Beschwerden nicht nur sprachlich oder mimisch. Sie kommunizieren ihr Leid auch über ihre Körpersprache. Dabei zeigen schmerzassoziierte Körperhaltungen spezifische klinische Muster. Diese signalisieren der Umwelt nicht nur das Vorhandensein von Schmerz, sondern kommunizieren auch dessen Art, Lokalisation und Intensität. Gerade bei sprachlich eingeschränkten Menschen dient dieser posturale Kommunikationskanal als wichtiges diagnostisches Werkzeug.

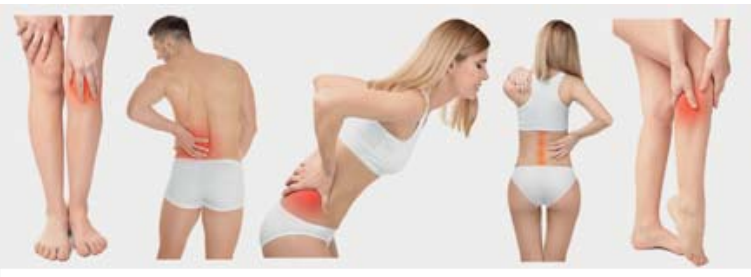

Quelle: Africa Studio/stock.adobe.com (Symbolbild).
Schmerz ist nicht nur eine sinnliche Erfahrung, sondern auch ein sozial-kommunikatives Ereignis. In diesem Sinne ist die Fähigkeit eines Menschen, Informationen über seine Schmerzen an andere Personen weiterzugeben, quasi der Schlüssel, damit er von diesen unterstützt, behandelt und sein Schmerz gelindert wird [1]. Im klinischen Rahmen kann diese Fähigkeit genutzt werden, um über eine effektive Schmerzkommunikation und -erkennung schneller zu einer genaueren Diagnose zu kommen und den Weg für eine wirksame Therapie zu ebenen [2].

Informationsquellen Obwohl die meisten Diagnosetechniken für Schmerzen auf der verbalen Berichterstattung im Rahmen der Anamnese beruhen, bieten nonverbal vom Patienten kommunizierte Hinweise wie seine Mimik und Körperhaltung eine umfangreiche Informationsquelle in Bezug auf Schmerzart, -intensität und -ort [3]. Dementsprechend können Ärzte, Physiotherapeuten und Pflegekräfte das nonverbale Verhalten eines Patienten bewusst verwenden, um so die Qualität ihrer Diagnose zu verbessern. Diesbezüglich gibt es sogar Hinweise, dass nonverbale Verhaltensweisen bisweilen eine genauere Quelle für diagnostische Informationen bei Schmerzpatienten darstellen [4].
Kommunikationskanal Die posturale Haltung ist somit ein wichtiger Kanal der nonverbalen Kommunikation, welcher zahlreiche Daten zur Unterstützung der Diagnose liefern kann. Die Entwicklung eines umfassenden Verständnisses der Art und Weise, wie sich Schmerzen durch die Körperhaltung ausdrücken, kann eine effektive klinische Praxis unterstützen.

\section{„Wenn die Worte fehlen, hilft ein Blick auf die Körpersprache.“}

\section{Körperhaltungen bei Schmerz}

Um die Körperhaltung als diagnostisches Kriterium zu nutzen, ist zunächst eine klare Definition der „schmerztypischen Körperhaltung“ erforderlich. Während die Schmerzkommunikation über nonverbale Kanäle wie die Mimik oder Lautäußerungen bereits gut erforscht und etabliert ist, gibt es dagegen einige Schwierigkeiten bei der Begriffsbestimmung archetypischer schmerzassoziierter Veränderungen der Körperhaltung [5-9]. Warum eine solche Definition so schwer zu formulieren ist, liegt v. a. in der Variabilität der Körperhaltung begründet. 
Variabilität Der menschliche Körper kann zwar als wirksames Kommunikationsmedium dienen, er wird jedoch selten ausschließlich zu diesem Zweck eingesetzt. So mag ein Individuum über seine Körperhaltung diverse Informationen kommunizieren, gleichzeitig wird er aber auch Teile seines Körpers für andere Aktivitäten verwenden - die unteren Extremitäten etwa für das Gehen oder die Arme und Hände für Interaktionen mit Objekten. Aufgrund dieser Komplexität sind kommunikative Körperhaltungen nur selten so klar zu dekodieren wie Gesichtsausdrücke oder Lautäußerungen, wo der komplette Kommunikationskanal auf einen einzigen Kommunikationszweck ausgerichtet ist.

Vorteile Trotz dieser Variabilität bietet die Kommunikation über die Körperhaltung eine Reihe von Vorteilen gegenüber anderen nonverbalen Kanälen. Erstens bietet der Körper quasi eine größere „Leinwand“ für seine Umwelt: er ist von weithin sichtbar und ermöglicht die Kommunikation über weitaus größere Distanzen als allein die Mimik. Zweitens können bei besonderer Schmerzrelevanz die Veränderungen der Körperhaltung nicht nur das Vorhandensein von Schmerz signalisieren, sondern auch deren Art und Ort kommunizieren - nonverbale Informationen, die nicht durch den Gesichtsausdruck vermittelt werden können.

\section{Körperhaltungsbasiertes Schmerzverhalten}

Wenngleich bis dato kein archetypischer Schmerzausdruck in Bezug auf die Körperhaltung definiert wurde, ist v. a. eine Reihe gut etablierter Verhaltensweisen stark mit dem Phänomen Schmerz verbunden. Bereits im Jahre 1985 definierten Dennis C. Turk und Kollegen zwei körperhaltungsbasierte Schmerzverhalten: die Schutzhaltung sowie die Veränderung des Gangbildes [10].

\section{Schutzhaltung}

Eine schmerzassoziierte Körperhaltung dient dem Schutz einer Verletzung. Demgemäß wird bspw. ein verletzter Arm gestützt und der Körper dahingehend ausgerichtet, dass die Verletzung nicht in die Nähe potenzieller Kontakt- bzw. Gefahrenquellen gelangt ( $\mathbf{A} \mathbf{b b}$. 1).

\section{Änderungen des Gangbildes}

Veränderungen im Gangbild können ebenfalls auf Schmerzen hinweisen. Zu diesen Abweichungen gehören neben offensichtlichen Anzeichen wie dem Hinken auch subtile Veränderungen des Gehens wie langsames Bewegen oder eine gebückte Haltung.

Variationen der Haltungsmuster Diese beiden angeführten Schmerzverhaltensweisen bilden gemeinsam unterschiedlich ausgeprägte Haltungsmuster, die bei einer Reihe von Schmerzarten beobachtet wurden. Sie imponieren hauptsächlich mit einem Abweichen bzw. Wegneigen von der vertikalen Körperebene, einer gebeugten Haltung beim Stehen oder Sitzen sowie einer Verände-

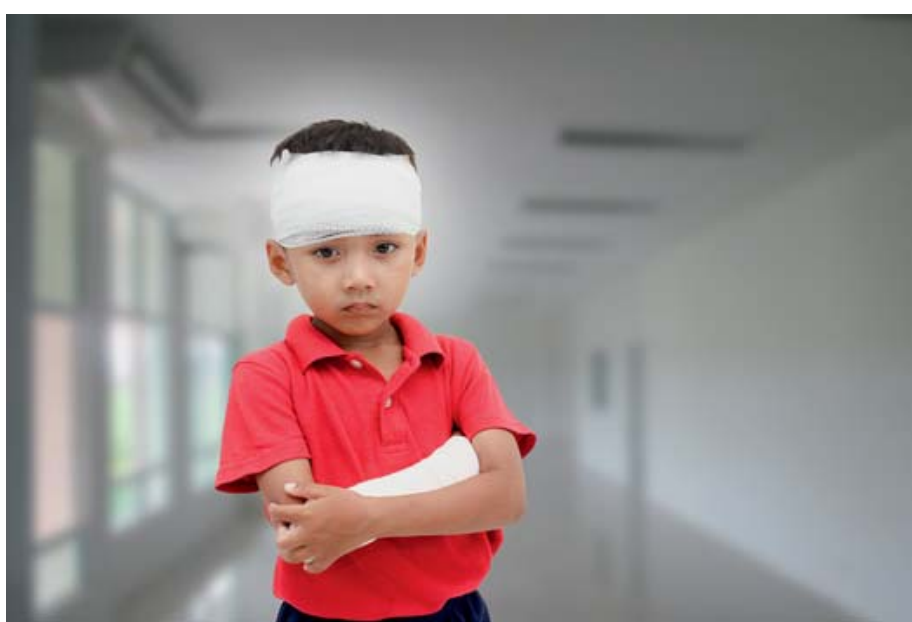

- Abb. 1 Bereits Kleinkinder zeigen ein körperhaltungsbasiertes Schmerzverhalten: der verletze Arm wird unterstützt und nah am Körper gehalten, um ihn vor möglicherweise schmerzhaften Kontakten zu schützen. Quelle: saiyood/stock.adobe.com (Symbolbild).

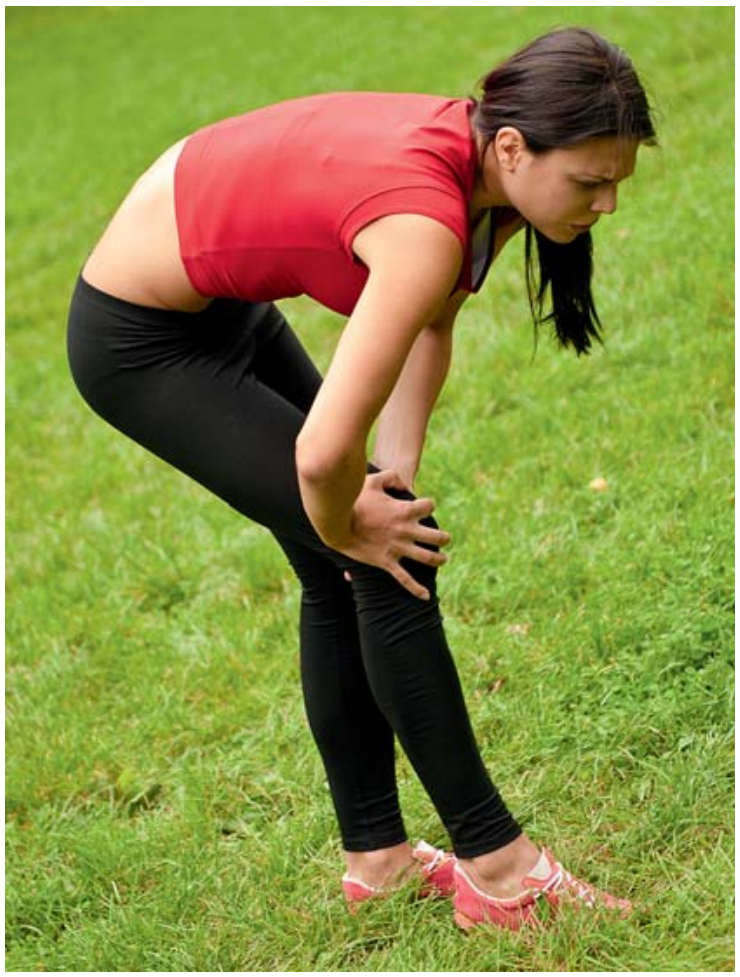

- Abb. 2 Ein verlangsamtes Schritttempo sowie eine gebückte Haltung können Indizien für Schmerzen sein. Quelle: Martinan/stock.adobe.com (Symbolbild).

rung der Beinposition, welche ein schlechteres Balanceverhalten sowie einen reduzierten Bewegungsspielraum zur Folge hat [11] ( A Abb. 2). 


\section{Körperhaltung als}

\section{Kommunikationsinstrument}

Die größte Evidenz in Bezug auf die Körperhaltung bei Schmerz fokussiert v.a. auf schmerzbedingte Veränderungen der Haltung sowie auf Schutzmechanismen. Darüber hinaus ist die Körperhaltung ein wichtiges Kommunikationsinstrument: Schmerzpatienten nehmen unterschiedliche Körperhaltungen nicht nur deshalb ein, um sich zu schützen und ihre Schmerzen zu lindern; sie versuchen gleichzeitig, ihre Beobachter über ihre Beschwerden zu informieren, um so eine Behandlung und andere unterstützende Interventionen zu ermöglichen und zu erleichtern.

\section{Signalisieren von Schmerzinformationen}

Diese sekundäre kommunikative Funktion der posturalen Schmerzverhaltensweisen muss nicht zwangsläufig vom Patienten beabsichtigt sein. Wie Arnstein Finset und Lidia Del Piccolo bereits im Jahre 2011 betonten ist v. a. wichtig, dass die Beobachter den Sinn dieser Körperhaltungen und Gesten erkennen können [12].

\section{Kommunikative Qualität}

Hierzu untersuchten Joseph Walsh, Christopher Eccleston und Edmund Keogh den kommunikativen Wert von Schmerzkörperhaltungen [13]. Sie präsentierten ihren Probanden verschiedene Körperhaltungen bei Schmerz sowie bei anderen emotionalen Zuständen. Obwohl die Körperhaltungen bei Schmerz unterschiedlicher ausgeprägt und somit gewissermaßen schwerer zu dekodieren waren als jene bei Emotionen wie Wut oder Freude, lag die Erkennungsgenauigkeit für Schmerzkörperhaltungen etwas höher als jene für andere postural ausgedrückten Gefühle (94\% vs. 92\%).

\section{Merke}

Schmerzassoziierte Körperhaltungen

Schmerzpatienten imponieren konsistent mit diversen Kennzeichen ihrer Körperhaltung. Folgende Merkmale können von Physiotherapeuten mit einem hohen Grad an Genauigkeit beobachtet und für Diagnostik und Therapie genutzt werden:

- Schutzverhalten: Abwenden von Rumpf und Kopf gegenüber Stimuli

- Handkontakt mit potenziell verletzten Körperstellen

- gebeugte Haltung bzw. Beugung von Knien und Hüften [11]

\section{Geschlechtsspezifische Unterschiede}

Später durchgeführte Folgeuntersuchungen zeigten, dass es geschlechtsspezifische Unterschiede in der Art und Weise geben könnte, wie Menschen die Schmerzkommunikation wahrnehmen. Ein besonderes Augenmerk der Forschung wird auf das weibliche Geschlecht gelegt: Frauen kommunizieren Schmerzen intensiver als

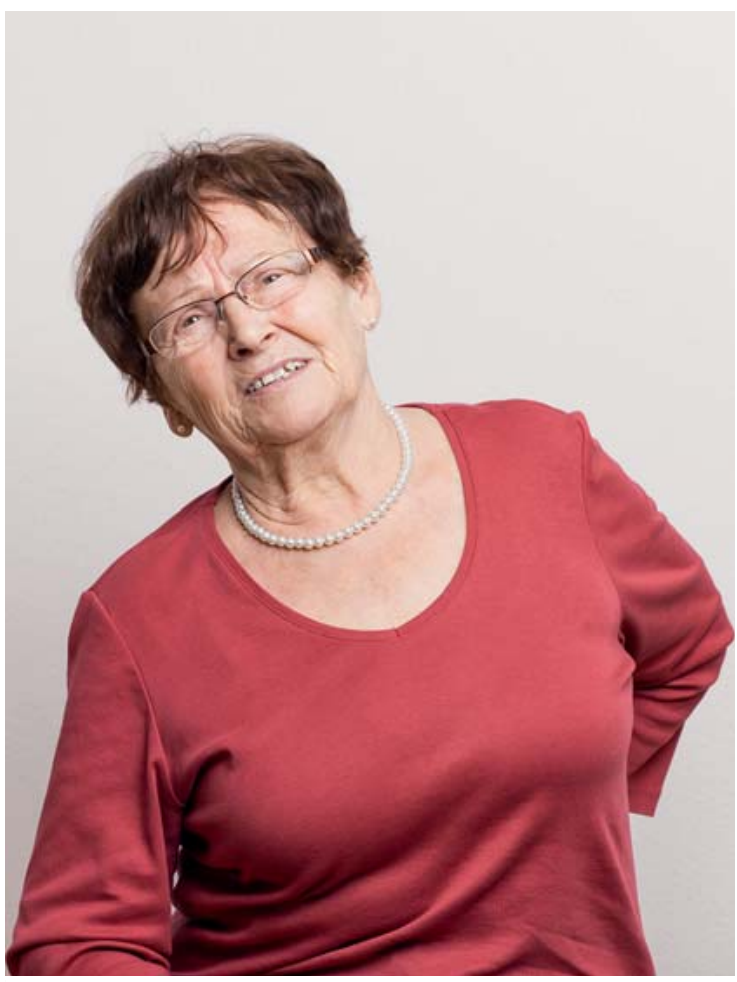

- Abb. 3 Nonverbale Schmerzsignale können besser identifiziert werden, wenn sie sowohl in der Körperhaltung apparent werden, als auch synchron durch die Mimik kommuniziert werden.

Quelle: DDRockstar/stock.adobe.com (Symbolbild).

Männer [14]. Dieser Umstand mag zum Teil für die unterschiedliche Diagnose und Behandlung von weiblichen und männlichen Patienten verantwortlich sein [15-17].

\section{Kombination nonverbaler Informationsquellen}

Durch die Körperhaltung können Schmerzen zwar effektiv kommuniziert werden, doch das alleinige Berücksichtigen dieser posturalen Indizien für das Erkennen von Schmerzen und deren Diagnose hat Grenzen. Entsprechend wichtig ist es, Informationen aus mehreren nonverbalen Kanälen zu kombinieren, um auf diesem Weg die Qualität der Schmerzdiagnostik zu verbessern.

Haltung und Mimik Zu diesem Punkt stellten Hillel Aviezer et al. fest, dass sich die Fähigkeit eines Beobachters bzgl. der Differenzierung zwischen ähnlichen emotionalen Zuständen wie bspw. Traurigkeit und Schmerz verbessert, wenn ihm simultan der Gesichtsausdruck und die Körperhaltung präsentiert werden [18]. Demzufolge ist es wichtig, dass ein klinisches Assessment mehrere nonverbale Kanäle berücksichtigt ( $\triangleright$ Abb. $\mathbf{3}$ ). 


\section{Merke}

Körperhaltung und Diagnostik

Die Körperhaltung besitzt einen hohen kommunikativen Wert und ist dementsprechend ein wichtiges klinisch-diagnostisches Instrument. Mithilfe dieses Werkzeugs können Physiotherapeuten die Schmerzen eines Patienten anhand dessen posturaler $\mathrm{Hal}$ tung erkennen und angemessen darauf reagieren.

\section{Klinische Instrumente}

Angesichts der experimentellen Beweise, dass die Körperhaltung eines Menschen genaue, zuverlässige und v.a. sehr gut erkennbare Informationen über dessen Schmerzen an den Beobachter übermittelt, ist es nicht verwunderlich, dass das Assessment posturaler Merkmale ein wichtiger Bestandteil der Diagnose in der klinischen Schmerzpraxis ist. Dies führte zur Entwicklung einer Reihe von Instrumentarien, bei denen die Körperhaltung als zentrales diagnostisches Kriterium dient.

\section{Patientengruppen}

Diagnostische Bewertungsinstrumente, die nonverbales Verhalten einschließlich der Mimik und der Körperhaltung zentralisieren, sind gerade bei folgenden Patientengruppen mit eingeschränkter verbaler Kommunikationsfähigkeit besonders wichtig:

- Neugeborene [19]

- Kinder $[20,21]$

- Patienten mit Störungen, welche die verbale Kommunikation beeinträchtigen (bspw. Demenz) [22]

AGS-Kriterien Zur Beurteilung älterer Demenzpatienten ist das nonverbale Schmerzverhalten so immanent wichtig, dass sich drei der sechs von der ,American Geriatric Society، (AGS) definierten Schmerzbeurteilungskriterien allein auf nonverbale Hinweise konzentrieren. In den AGS-Kriterien werden besonders die Körperhaltung sowie das Gangbild hervorgehoben [23].

\section{Kombination von Haltung und Mimik}

Die Mehrzahl der Instrumentarien zur Beurteilung von Haltungsmerkmalen in der klinischen Schmerzpraxis basiert auf einer Kombination verschiedener Assessments und berücksichtigt hierzu unterschiedliche nonverbale Quellen [24]. Die Forschung hat gezeigt, dass dieser Ansatz die genaueste und über die Zeit stabilste Beurteilung der Schmerzintensität liefert [25].

Weil die klinischen Instrumentarien spezifische Schmerztypen unterschiedlich bewerten, ist es sehr wichtig sicherzustellen, dass die in der Praxis angewendeten Instrumentarien konsequent und exakt auf bestimmte Patientengruppen zugeschnitten sind [26].

\author{
Merke \\ Nonverbale Schmerzevaluation \\ Folgende Verhaltensweisen von Schmerzpatienten \\ erscheinen - ungeachtet der Variationen klinischer \\ Instrumentarien und über alle Messverfahren hinweg \\ - konsistent für eine nonverbale Schmerzevaluation: \\ - Muskelverspannungen \\ - Schutzverhalten \\ - schmerzassoziierte Veränderungen \\ der Gesichtsmimik
}

\section{Autonomie nonverbaler Schmerzausdrücke}

Expressionsmechanismen Ein wesentlicher Vorteil bei der Verwendung nonverbaler Schmerzausdrücke in der Diagnostik besteht darin, dass sie als autonom angesehen werden können. Diesbezüglich unterschieden Kenneth Craig et al. zwischen autonomen und kontrollierten Expressionsmechanismen [27]. Sie schlugen vor, dass nonverbale Expressionsmechanismen in der Regel autonomer seien und - gewissermaßen „aus dem Bauch heraus“ - die Intensität und die Art des Schmerzes genauer widerspiegeln könnten. Überdies bewirken autonome schmerzassoziierte Haltungsmuster eines Patienten intuitiv stärkere neurologische Reaktionen bei seinem Beobachter [28]. Im Gegensatz hierzu erfordert die kontrollierte verbale Berichterstattung eines Individuums über seine Schmerzen von diesem eine stärkere kognitive Kontrolle ( $\triangleright$ Abb. 4 ).

Unterschätzungs-Bias Gleichzeitig kann das effektive Berücksichtigen nonverbaler Schmerzhinweise bei der Diagnose die Tendenz zur irrtümlichen Unterschätzung der Schmerzen reduzieren [29]. Ein entsprechendes Fachwissen von Klinikern kann folglich die Genauigkeit von nonverbalen Schmerzbewertungen erhöhen [30].

Und schließlich ist das Unterdrücken nonverbaler Schmerzhinweise bei einem Patienten leichter zu erkennen als dessen ungenaue bzw. lückenhafte verbale Berichterstattung im Rahmen der Anamnese [31].

\section{Fazit}

Schmerzkörperhaltungen dienen einem wichtigen kommunikativen Zweck. Durch sie können Physiotherapeuten Schmerzen früher erkennen und schneller adäquat reagieren. Gerade bei Neonaten, Kindern und Demenzkranken sind schmerzassoziierte Körperhaltungen im klinischen Setting ein wichtiges diagnostisches Instrument, um deren Schmerzart und -intensität exakt beurteilen zu können. Werden sie mit weiteren diagnostischen Maßnahmen wie der Anamnese sowie anderen nonverbalen Informationsquellen wie der Mimik kombiniert, bietet dies die genaueste und über die Zeit stabilste Beurteilung der Schmerzintensität. 

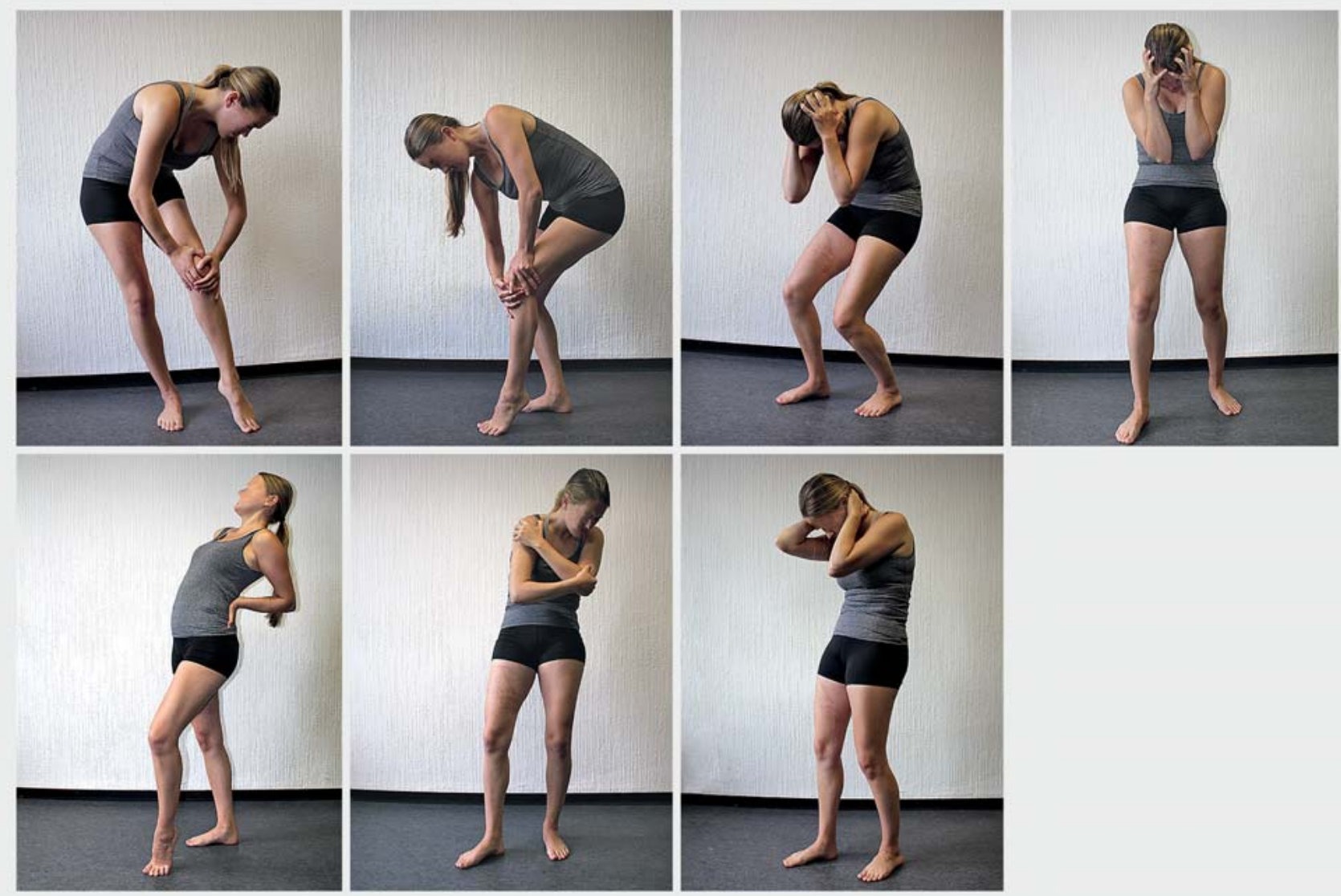

- Abb. 4 Klassische schmerzassoziierte Körperhaltungen erfolgen autonom und können vom Patienten kaum kontrolliert werden. Quelle: Harry von Piekartz.

Ausblick Während die meiste Evidenz zur nonverbalen Schmerzkommunikation bisher zur En- und Dekodierung von mimisch kommunizierten Schmerzen vorliegt, sind schmerzassoziierte Körperhaltungen relativ wenig erforscht. Zwar drücken sich Schmerzen erwiesenermaßen durch die Körperhaltung aus - sie imponieren mit Schutzverhalten, Gangstörungen und Muskelverspannungen und werden von den Patienten quasi autonom nach außen kommuniziert, aber die Forschung zur Variation der Schmerzexpression zwischen Schmerztypen und -zuständen bleibt unterentwickelt und sollte intensiver betrachtet werden. Entsprechend sind weitere Untersuchungen nötig, um eine vollständige und differenzierte Darstellung schmerzassoziierter Körperhaltungen zu gewährleisten und hieraus ein Verständnis dafür zu entwickeln, wie der menschliche Körper Schmerzinformationen kommuniziert.
Autorinnen/Autoren

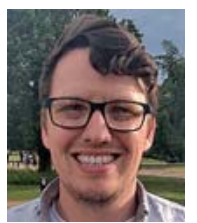

Dr. Joseph Walsh ist Dozent für Psychologie an der Bath Spa University in England. Seine Forschung konzentriert sich auf experimentelle Arbeiten, in denen untersucht wird, wie Schmerz durch die Körperhaltung kommuniziert wird, sowie auf Aufmerksamkeitsprozesse, welche bei der Erkennung von Verhaltensweisen von Haltungsschmerzen eine Rolle spielen.

Korrespondenzadresse

\section{Dr. Joseph Walsh}

Senior Lecturer in Psychology

Bath Spa University

Newton Park

Newton St Loe

GB-Bath BA2 9BN

J.walsh2@bathspa.ac.uk 
[1] Craig KD. The social communication model of pain. Canadian Psychology/Psychologie canadienne 2009; 50 (1): 22-32. doi:10.1037/a0014772

[2] Herr K, Coyne PJ, Key T et al. Pain assessment in the nonverbal patient: position statement with clinical practice recommendations. Pain Manag Nurs. 2006; 7 (2): 44-52. doi:10.1016/j. pmn.2006.02.003

[3] de Andrade DC, de Faria JWV, Caramelli P et al. The assessment and management of pain in the demented and nondemented elderly patient. Arquivos de Neuro-Psiquiatria 2001; 69 (2-B): 387-394. doi:10.1590/50004-282X2011 000300023

[4] Prkachin KM, Schultz IZ, Hughes E. Pain Behavior and the Development of Pain-related Disability: The Importance of Guarding. Clin J Pain 2007; 23 (3): 270-277. doi:10.1097| AJP.0b013e3180308d28

[5] LeResche L, Dworkin SF. Facial expressions of pain and emotions in chronic TMD patients. Pain, 1988; 35 (1): 71-78. doi:10.1016/0304-3959(88)90278-3

[6] Prkachin KM, Solomon PE. The structure, reliability and validity of pain expression: Evidence from patients with shoulder pain. Pain 2008; 139 (2): 267-274. doi:10.1016/j.pain.2008.04.010

[7] Belin P, Fillion-Bilodeau S, Gosselin F. The Montreal Affective Voices: A validated set of nonverbal affect bursts for research on auditory affective processing. Behavior Research Methods 2008; 40 (2): 531-539. doi:10.3758/brm.40.2.531

[8] Coulson M. Attributing emotion to static body postures: recognition accuracy, confusions, and viewpoint dependence. Journal of Nonverbal Behavior 2004; 28 (2): 117-139. doi:10.1023/B:JONB.0000023655.25550.be

[9] de Gelder B. Towards the neurobiology of emotional body language. Nature Reviews Neuroscience 2006; 7 (3): 242-249. doi:10.1038/nrn1872

[10] Turk DC, Wack JT, Kerns RD. An empirical examination of the "pain-behavior" construct. Journal of Behavioral Medicine 1985; 8 (2): 119-130. doi:10.1007/bf00845516

[11] Haugstad GK, Haugstad TS, Kirste UM. Posture, movement patterns, and body awareness in women with chronic pelvic pain. Journal of Psychosomatic Research 2006; 61 (5): 637644. doi:10.1016/j.jpsychores.2006.05.003

[12] Finset A, Piccolo LD. Nonverbal communication in clinical contexts. In: Rimondi M, Hrsg. Communication in cognitive behavioral therapy. New York: Springer Science + Business Media; 2011

[13] Walsh J, Eccleston C, Keogh E. Pain communication through body posture: The creation and validation of a stimulus set. Pain 2014; 155 (11): 2282-2290. doi:10.1016/j.pain.2014. 08.019

[14] Walsh J, Eccleston C, Keogh E. Sex differences in the decoding of pain-related body postures. European Journal of Pain 2018; 21 (10): 1668-1677. doi:10.1002/ejp.107

[15] Fillingim RB, King CD, Ribeiro-Dasilva MC et al. Sex, Gender, and Pain: A Review of Recent Clinical and Experimental Findings. The Journal of Pain 2009; 10 (5): 447-485. doi:10.1016/j. jpain.2008.12.001

[16] Levine FM, de Simone LL. The effects of experimenter gender on pain report in male and female subjects. Pain 1991, 44 (1): 69-72. doi:10.1016/0304-3959\%2891\%2990149-R

[17] Rosseland LA, Stubhaug A. Gender is a confounding factor in pain trials: women report more pain than men after arthroscopic surgery. Pain 2004; 112 (3): 248-253. doi:10.1016/j. pain.2004.08.0289

[18] Aviezer H, Trope Y, Todorov A. Body cues, not facial expressions, discriminate between intense positive and negative emotions. Science 2012; 338 (6111): 1225-1229. doi:10.1126/science. 1224313

[19] Hand IL, Noble L, Geiss D et al. COVERS Neonatal Pain Scale: Development and Validation. International journal of pediatrics; 2010: 496719. doi:10.1155/2010/496719

[20] McGrath P], Johnson G, Goodman JT et al. CHEOPS: a behavioral scale for rating postoperative pain in children. Advances in Pain Research and Therapy 1985; 9: 395-402

[21] Tarbell SE, Thomas Cohen I, Marsh JL. The Toddler-Preschooler postoperative pain scale: an observational scale measuring postoperative pain in children aged 1-5. Preliminary report. Pain 1992; 50 (3): 273-280. doi:10.1016/0304-3959(92) 90031-6

[22] Herr K, Bjoro K, Decker S. Tools for Assessment of Pain in Nonverbal Older Adults with Dementia: A State-of-the-Science Review. Journal of Pain and Symptom Management 2006; 31 (2): 170-192. doi:10.1016/j.jpainsymman.2005.07.001

[23] Hadjistavropoulos T, Fitzgerald TD, Marchildon GP. Practice guidelines for assessing pain in older persons with dementia residing in long-term care facilities. Physiotherapy Canada 2010; 62 (2): 104-113. doi:10.3138/physio.62.2.104

[24] Jensen MP, Turner LR, Turner JA et al. The use of multiple-item scales for pain intensity measurement in chronic pain patients. Pain 1996; 67 (1): 35-40. doi:10.1016/0304-3959(96)03078-3

[25] Jensen MP, Turner JA, Romano JM et al. Comparative reliability and validity of chronic pain intensity measures. Pain 1999; 83 (2): 157-162. doi:10.1016/S0304-3959(99)00101-3

[26] Lints-Martindale AC, Hadjistavropoulos T, Lix LM et al. A comparative investigation of observational pain assessment tools for older adults with dementia. Clin J Pain 2012; 28 (3): 226237. doi: $10.1097 / A J P .0 b 013 e 3182290 \mathrm{~d} 90$

[27] Craig KD, Versloot J, Goubert L et al. Perceiving pain in others: automatic and controlled mechanisms. J Pain 2010; 11 (2): 101-108. doi:10.1016/j.jpain.2009.08.008

[28] Botvinick M, Jha AP, Bylsma LM et al. Viewing facial expressions of pain engages cortical areas involved in the direct experience of pain. Neurolmage 2005; 25 (1): 312-319. doi:10.1016/j. neuroimage.2004.11.043

[29] Prkachin KM, Solomon PE, Ross J. Underestimation of pain by health-care providers: towards a model of the process of inferring pain in others. Can J Nurs Res. 2007; 39 (2): 88-106

[30] Cheng Y, Lin CP, Liu HL et al. Expertise modulates the perception of pain in others. Curr Biol. 2007; 17 (19): 1708-1713. doi:10.1016/j.cub.2007.09.020

[31] Craig KD, Hyde SA, Patrick CJ. Genuine, suppressed and faked facial behavior during exacerbation of chronic low back pain. Pain 1991; 46 (2): 161-171

Bibliografie

DOI https://doi.org/10.1055/a-0971-0390

Der Schmerzpatient 2019; 2: 152-157

(c) Georg Thieme Verlag KG Stuttgart · New York ISSN 2512-6210 